\title{
Die Schweiz ist nicht knuffig
}

\section{Matthias Scholer}

Online- und Printredaktor SÄZ

\begin{abstract}
Rund ein Drittel der Ärztinnen und Ärzte in der Schweiz kommt aus dem Ausland. Weshalb haben sie sich für ein Leben hierzulande entschieden? Welches sind kulturelle Stolpersteine, die es zu umschiffen gilt? Und was halten sie von der Schweizer Gesundheitspolitik? Diesen und weiteren Fragen möchten wir in der neuen, in loser Folge erscheinenden Artikelserie «Grüezi Schweiz» nachgehen. In dieser Ausgabe stellen wir Stefan Graf vor, der vor bald 20 Jahren den Sprung in die Schweiz wagte und sich hier nun zu Hause fühlt - auch wenn der Start nicht ganz reibungslos verlief.
\end{abstract}

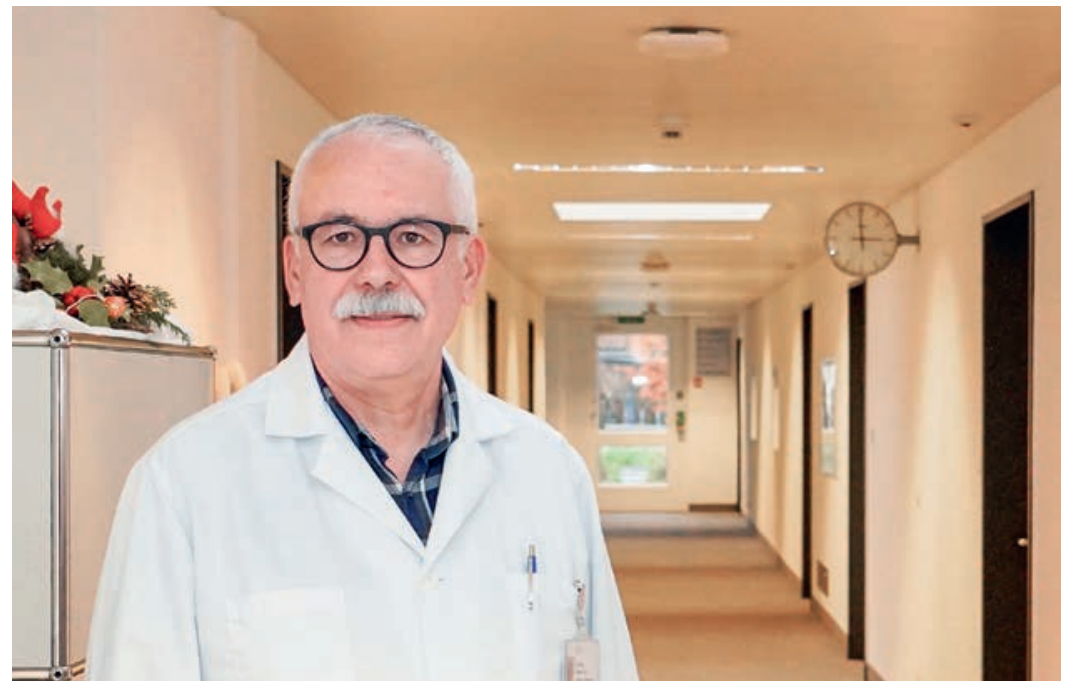

Stefan Graf passte sich auch sprachlich der Schweiz an.

Für Schweizer Ärztinnen und Ärzte war die vakante Stelle wohl nicht attraktiv genug. Während Monaten meldete sich niemand auf ein Inserat des Bezirksspitals im freiburgischen Tafers. Gesucht waren zwei Fachärzte, die sich in einer Co-Funktion die Chefarztposition Anästhesie teilten. Folglich wurde die Stelle auch im Deutschen Ärzteblatt ausgeschrieben. Stefan Graf sah darin gleich eine Chance. Denn: «Ohne Habilitation hat man in Deutschland als Facharzt kaum eine Möglichkeit, in eine Kaderposition aufzusteigen.» Mit der Bewerbung klappte es reibungslos, und Graf zog von Freiburg im Breisgau mit Kind und Kegel in die Schweiz. Das war 2002.

Heute weiss Stefan Graf, dass dieser Schritt weder für ihn noch für seine Familie ganz so einfach war, wie anfänglich gedacht, und einigen Kollateralschaden auf privater Seite verursachte. Einerseits bedeutete die
Co-Funktion nämlich eine enorme Arbeitsbelastung: "Jede zweite Nacht und jedes zweite Wochenende hatte ich Dienst.» Andererseits war es speziell für seine Kinder schwer, in dem ländlich geprägten Tafers Fuss zu fassen. Heute würde man wohl von Mobbing sprechen. Die Kinder wurden auf Grund ihrer Herkunft gehänselt und ausgegrenzt. Grafs Sohn trat die Flucht nach vorne an: «Er zog gleich nach der obligatorischen Schulzeit nach Basel, um eine Lehre zu absolvieren. Heute lebt er in Neuseeland.» Die Tochter zog später ebenfalls weg, wählte jedoch während ihrer Schulzeit eine andere Überlebensstrategie: "Sie lernte innert Kürze Senslerdeutsch und konnte sich so dem Spott ihrer Mitschüler entziehen.»

\section{Ein linguistisches Chamäleon}

Die Fähigkeit der Tochter, den exotischen Dialekt bis zur Perfektion zu erlernen, kommt wohl von Stefan Grafs Seite. Denn auch er spricht astrein Schweizerdeutsch - sogar mit einem unüberhörbaren freiburgerischen Einschlag. «Mir fällt es leicht, die Tonalität einer Sprache zu übernehmen. Als ich als Assistenzarzt ein Jahr lang in Irland arbeitete, glaubten danach viele, dass ich von der Grünen Insel stamme», erzählt der Mediziner lachend. Diese sprachliche Anpassungsfähigkeit erlaubt Graf, dem Schubladendenken seiner Mitmenschen ein Schnippchen zu schlagen: «Merkt das Gegenüber erst nach einiger Zeit, dass man gar kein 'Hiesiger' ist, ist es für Vorurteile zu spät. Man hat sich ja bereits kennengelernt.»

Beruflich lief bei Graf alles rund. Was ihn am meisten motivierte, war die Tatsache, dass er und sein Kollege dank den schlanken Strukturen des Bezirksspitals viel verwirklichen und so über die Jahre die Anästhesio- 
logie zu einer «tollen Abteilung» ausbauen konnten, die sich vor keinem Vergleich mit grossen Spitälern zu fürchten brauchte.

Doch dann geriet auch das kleine Spital in Tafers in den Strudel der Spitalfusionen: Das Kantonsspital Freiburg übernahm den Lead. «Dabei ging vieles kaputt, was wir über die Jahre aufgebaut hatten", blickt Stefan Graf mit einer gewissen Ernüchterung zurück. 2014 zog der Anästhesist die Konsequenzen und wechselte ins Regionalspital Münsingen. Hier arbeitet Graf nicht mehr als Chefarzt, sondern als Leitender Arzt Anästhesie. Trotzdem ist er mit seiner Stelle zufrieden: «Mit meinen 60 Jahren bin ich nicht traurig, seltener Dienste zu schieben.» Zudem müsse er an weniger Sitzungen teilnehmen und habe so mehr Zeit für die Medizin.

\section{Ein Spital ist keine Autofabrik}

Stefan Graf ist definitiv in der Schweiz angekommen, und er plant auch nicht, in sein Heimatland zurückzukehren. Ein Grund dafür sind die besseren Arbeitsbedingungen. Diese sieht Graf jedoch in Gefahr, sollte die Schweiz weiterhin ziemlich unkritisch Konzepte aus dem Ausland übernehmen, bei denen die Erfahrungen zeigen, dass sie nicht funktionieren. «Wenn meine deutschen Kollegen hören, dass wir uns hier ernsthaft überlegen, Globalbudgets einzuführen, verstehen sie die Welt nicht mehr», erzählt Stefan Graf.

Und er fügt an: «Weltweit werden die Gesundheitswesen von ökonomischen Überlegungen dominiert. Ein Spital ist doch keine Autofabrik. Da funktioniert die Managerlogik nicht.» Der Deutsche bedauert zudem, dass die Gesundheitswesen fast ausschliesslich von der Kosten- und nie von der Leistungsseite betrachtet werden. Dabei seien doch die Fortschritte im Therapiebereich enorm und die volkswirtschaftliche Bedeutung der Gesundheitsbranche bedeutend.

\section{Der Röstigraben lebt}

In Grafs Augen nutzen wir hierzulande einen weiteren Vorteil zu wenig: die kulturellen Unterschiede. «Die französischsprachigen Kolleginnen gehen für Weiterbildungen nach Frankreich, die deutschsprechenden Kollegen nach Deutschland und die italienischsprechenden nach Italien", fasst Stefan Graf seine Beobachtungen zusammen. Damit profitieren die in der Schweiz tätigen Ärztinnen und Ärzte vom Know-how führender Universitätskliniken verschiedener Länder. Würde dieses diversifizierte Wissen miteinander geteilt, hätte man gegenüber anderen Nationen einen weiteren Standortvorteil. Aber: «Häufig sprechen die zugewanderten Mediziner nur eine Landessprache. Dazu kommt der Reflex, dass man sich lieber abschottet, wenn das Gegenüber nicht dieselbe Sprache spricht, anstatt einen Weg zu finden, den sprachlichen Röstigraben zu überwinden und die verschiedenen Erfahrungen auszutauschen.»

\section{Sprachliche Eigenheiten übernehmen}

Und welches sind die grössten Stolperfallen für deutsche Kolleginnen und Kollegen, die sich in der Schweiz niederlassen wollen? «Man muss sich bewusst sein, dass man in ein fremdes Land auswandert. Viele meiner Landsleute machen den Fehler und sehen die Schweiz nur als romantisches Anhängsel Deutschlands. Einfach etwas 'heimeliger' mit knuffigen Menschen. Das ist natürlich Blödsinn", sagt Graf. Der Umstand, dass in der Deutschschweiz die Sprache dieselbe ist, sei einerseits ein Vorteil. Andererseits höre man dann als Deutscher auch nicht mehr genau hin und verpasse so sprachliche Feinheiten, die einen kleinen Kulturschock beim Gegenüber auslösen können. Dazu gehört zum Beispiel das Wort «bitte», das hierzulande viel häufiger eingesetzt wird. «In der Schweiz gibt es eine Form der Höflichkeit, die in Deutschland als altbacken gilt», gibt Graf zu bedenken. Und fügt an: «Ich persönlich empfinde diesen höflichen Umgang im Alltag als Mehrwert.»

Nur in einer Sache bleibt Graf seiner Linie treu: «Ich diskutiere im Team jedes Thema zu Ende und lasse nicht zu, dass etwas unter den Teppich gekehrt wird, nur weil man befürchtet, anzuecken.» Da hat auch ein typisch schweizerischer Kompromiss einen schweren Stand.

Bildnachweis

Matthias Scholer

Bisher in dieser Serie erschienen:

Licht und Schatten

Urologe Mario Shlomo Kuntze (2019/7)

"Niemand hat gedacht, dass ich bleiben werde»

Talärztin Lutgard Werlen (2019/1-2)

Ein Bayer in der Schweiz

Hausarzt Robert Klingl (2018/44)

Gekommen und geblieben

Hausärztin Kathleen Keilwerth (2018/23)

Für unsere Serie "Grüezi Schweiz» suchen wir ausländische Ärztinnen und Ärzte, die uns einen Einblick in ihr Leben und ihren Berufsalltag gewähren. Wir freuen uns über eine Kontaktaufnahme: mscholer[at]emh.ch 